\title{
Astragaloside II induces osteogenic activities of osteoblasts through the bone morphogenetic protein-2/MAPK and Smad1/5/8 pathways
}

\author{
XIANG-HE KONG $^{1}$, YIN-BO NIU ${ }^{1}$, XIAO-MEI SONG ${ }^{3}$, DONG-DONG ZHAO ${ }^{3}$, JUAN WANG ${ }^{1}$, \\ XIANG-LONG WU ${ }^{1}$, RONG ZHANG ${ }^{2}$ and QI-BING MEI ${ }^{1,2}$ \\ ${ }^{1}$ School of Life Sciences, Northwestern Polytechnical University, Shaanxi; ${ }^{2}$ Department of Pharmacology, \\ School of Pharmacy, The Fourth Military Medical University, Shaanxi; ${ }^{3}$ College of Pharmacy, \\ Shaanxi University of Chinese Medicine, Shaanxi, P.R. China
}

Received December 26, 2011; Accepted February 20, 2012

DOI: $10.3892 / \mathrm{ijmm} .2012 .941$

\begin{abstract}
Radix Astragalus has been identified to exert beneficial effects in preventing postmenopausal bone loss. However, the active ingredients and mechanism of action remain unknown. In this study, we examined the effect of Astragaloside II (AST II), which is a monomer of Astragalus saponin, on the viability, proliferation, differentiation and maturation of rat primary osteoblasts, as well as its relevant molecular mechanism. We found that AST II exhibits a significant induction of proliferation, differentiation and mineralization in primary osteoblasts. AST II stimulates osteoblast differentiation at various stages, from early to late stage of differentiated osteoblasts. Furthermore, induction of differentiation by AST II is associated with increased expression of bone morphogenetic protein-2 (BMP-2), activation of Smad1/5/8, ERK1/2 and p38, and increased expression of core-binding factor 1 (Cbfa1)/Runx2. BMP antagonist (Noggin) blocks the effect of AST II on cell differentiation, and Smad1/5/8, p38, Cbfa1 expression, but only partly decreases ERK1/2 activation. This indicates that BMP-2 is essential in AST II-mediated osteoblast differentiation and Smad1/5/8, p38, Cbfa1 activation, and is partly involved in ERK1/2 activation. In conclusion, although in vivo studies are required in the future, as a phyto-saponin of Radix Astragalus, AST II may become a novel candidate that is beneficial for stimulating the osteoblastic activity resulting in bone formation, which has not been recognized and reported previously.
\end{abstract}

Correspondence to: Dr Qi-Bing Mei, School of Life Sciences, Northwestern Polytechnical University, Shaanxi 710072, P.R. China E-mail: qbmei@nwpu.edu.cn

Dr Rong Zhang, Department of Pharmacology, School of Pharmacy, The Fourth Military Medical University, Shaanxi 710032, P.R. China E-mail: hushijie@fmmu.edu.cn

Key words: Astragaloside II, osteoblasts, osteogenic activities, bone morphogenetic protein-2, Smad1/5/8, mitogen-activated protein kinases

\section{Introduction}

Osteoporosis is a reduction in skeletal mass due to an imbalance between bone resorption by osteoclasts and bone formation by osteoblasts. The formation of bone involves a complex series of events, including the proliferation and differentiation of osteoblasts and result eventually in the formation of a mineralized extracellular matrix $(1,2)$. Among cell signaling pathways associated with bone formation, bone morphogenetic proteins (BMPs) play important roles in bone formation and osteoblasts differentiation by stimulating alkaline phosphatase (ALP) activity and synthesis of proteoglycan, collagen, fibronectin and osteocalcin (3-5). In the BMP subfamily, BMP-2 has demonstrated a strong osteo-inductive capacity in vivo and in vitro $(6,7)$. It transduces signals through Smad-dependent and Smad-independent signalling pathways (8). BMP-2 stimulates the activation (phosphorylation) of Smad1/5/8 proteins $(9,10)$ and extracellular signal-regulated protein (ERK)1/2, p38, c-Jun kinases mitogen-activated protein kinases (MAPKs) $(9,11,12)$. Besides, the core binding factor (Cbfa1/Runx2) was reported as the most important transcription factor for osteoblastic differentiation (13). Both the Smad and MAPK pathways are essential components of the BMP signaling during osteoblast differentiation (11,14-16) and for Cbfa1 induction $(17,18)$. Cbfal is also a pivotal target of some anti-osteoporosis drugs $(17,19,20)$.

Patients or potential patients of osteoporosis need longterm treatment or prevention. Current drugs used to treat osteoporosis include bone resorption inhibitors, such as bisphosphonates, calcitonin, estrogen, and ipriflavone, and bone formation promoters, such as fluoride, PTH and PGE. However, what concerns people are side effects of the longterm use of these drugs. For instance, estrogen may cause a higher incidence of breast carcinoma, endometrial cancer, and cardiovascular disease (21). Besides, the high costs of some drugs are unacceptable for some osteoporosis patients, especially in developing countries. Considering the broad spectrum effect of osteoporosis in the medical system, and the defects of existing drugs, succedaneum such as traditional Chinese medicine may be a more acceptable and safer approach to 
prevent osteoporosis. Plant-derived medicines display less adverse effects and have been a part of traditional healthcare in China for thousands of years. Many of these possess antiosteoporotic activities $(22,23)$.

The famous traditional Chinese Medicine Radix Astragalus is the root of Astragalus membranaceus (Fisch.) Bge. Radix Astragalus has been used as one of the superior 'tonics' in Chinese medicine for thousands of years, and used as an approved drug in the modern Pharmacopoeia of the People's Republic of China. It is noteworthy that Astragalus is one of the most frequently used herbs in formulas that are prescribed for the treatment of osteoporosis in China, such as Astragalus Sanxian Decoction(24), Drynol Cibotinis (25), and Buzhongyiqi Decoction (26), indicating the activity of anti-osteoporosis. Based on both the in vitro (27) and in vivo studies (28), Radix Astragalus were proved to be able to inhibit bone resorption, stimulate bone formation, and prevent ovariectomy-induced osteoporosis. However, as most of anti-osteoporosis studies on Astragalus were focused on the crude extract, the constituents active against anti-osteoporosis are still unknown. No systematic studies have so far been carried out to evaluate the effects of the active ingredients of Astragalus on the proliferation and differentiation of osteoblasts, and mechanism of the action.

Saponins have been reported to induce osteoblast osteogenesis. Researches have mainly focued on Panax notoginseng (29) and Panax ginseng (30) saponins. Astragalus saponins are the main active constituents of Astragalus. Radix Astragalus is mild, long-term use without significant side effects on the human body, and has been added in diet as a tonic in China for thousands of years. Moreover, compared with the rare and precious medicinal herbs (Panax notoginseng and Panax ginseng), Astragalus is much cheaper because of the rich plant resources and thus more affordable for patients. Therefore, we are extremely interested to investigate whether Astragalus saponins could be new anti-osteoporosis natural products or lead compounds. Astragaloside II (AST II) is a (3b, 6a, 16b, 20R, 24S)-3-((2-O-Acetyl- $\beta$-D-xylopyranosyl) oxy)-20, 24-epoxy-16, 25-dihydroxy-9, 19-cyclolanostan-6-yl $\beta$-D-glucopyranoside (Fig. 1). In the present study, we examined the concentrationdependent effects of AST II on the osteogenic activities, including proliferation, differentiation, as well as mineralization of osteoblasts. In addition, to establish the potential mechanism involved in the osteoprotective effects of AST II, we assayed BMP-2, SMADs, MAPKs and Cbfa1 levels, which are strongly associated with the signaling pathways of osteogenesis.

\section{Materials and methods}

Reagents. AST II was purchased from Phytomarker Bio-Technology Co., Ltd. (China). Estradiol was purchased from Sigma (USA). Fetal bovine serum (FBS), minimal essential medium (MEM), were purchased from HyClone (USA). L-ascorbic acid, $\beta$-glycerophosphate and MTT (3-(4, 5-dimethylthiazol-2-yl)-2,5-diphenyltetrazolium bromide) were purchased from Sigma. Ethynyl deoxyuridine (EdU) DNA imaging kit was purchased from RiboBio Co., Ltd. (China). ALP activity assay kit was purchased from Nanjing Jiancheng Bioengineering Institute (China). Rat osteocalcin and collagen I ELISA kit was purchased from Biotech Co., Ltd. (China). Nuclear and cytoplasmic protein extraction kit

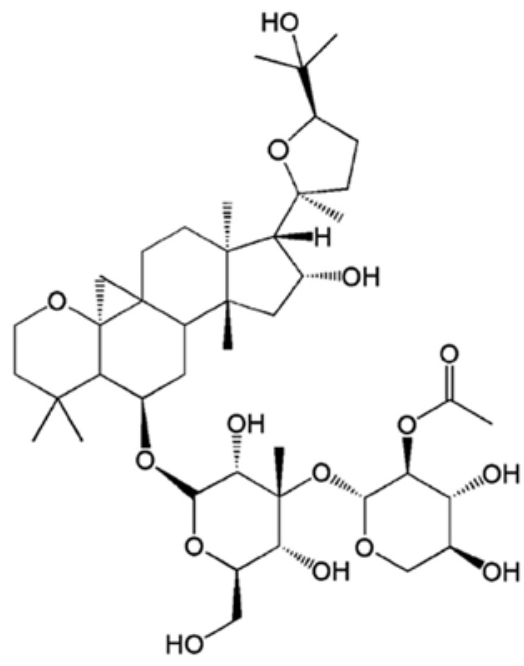

AST II structure

Figure 1. Structure of Astragaloside II (AST II).

and BCA-protein assay kit was purchased from Beyotime (Beyotime Institute of Biotechnology, Jiangsu, China). BMP-2 ELISA kit and noggin were purchased from R\&D Systems (USA). The antibodies to Smad1/5/8, p38, ERK1/2, JNK, phosphorylated Smad1/5/8, phosphorylated p38, phosphorylated ERK1/2, phosphorylated JNK were purchased from Cell Signaling (USA), Cbfa1, Histone and $\beta$-actin from Santa Cruz Biotechnology, Inc. (USA). The stock solution of AST II was prepared at a concentration of $0.02 \mathrm{M}$ of DMSO. It was then stored at $-20^{\circ} \mathrm{C}$ until use. For all experiments, the final concentrations of the test compound were prepared by diluting the stock with medium. Control cultures received the carrier solvent $(0.05 \%$ DMSO).

Animals. Newborn Sprague-Dawley rats were obtained from the Experimental Animal Center, the Fourth Military Medical University. Animal care and experiments were approved and conducted in accordance with accepted standards of animal care and use as deemed appropriate by the Ethics Committee for Animal Care and Use of the Fourth Military Medical University.

Isolation and culture of rat calvarial osteoblasts. The calvaria of fetal rats were dissected from fetal rats. Primary osteoblastic cells were prepared by the method as previously described (31). The cells were cultured in MEM, supplemented with $10 \%$ FBS and antibiotics (100 U/ml of penicillin G and streptomycin $100 \mathrm{mg} / \mathrm{ml}$ ) in a humidified atmosphere of $5 \%$ $\mathrm{CO}_{2}$ and $95 \%$ air, at $37^{\circ} \mathrm{C}$. The characteristics of osteoblasts were confirmed by morphology, the expression of alkaline phosphatase, and the formation of mineralized matrix. All animals were treated according to the Guide for Care and Use of Laboratory Animals with the approval of Institutional Ethics Committee of the Fourth Military Medical University on animal experiment.

Cell viability assay. Cell viability was determined by means of MTT assay. Primary osteoblastic cells were seeded into 96 -well plates at a density of $4 \times 10^{3}$ cells/well in full medium 
containing $10 \%$ FBS. After $24 \mathrm{~h}$, the cells were washed twice with PBS solution and then AST II was added at concentrations ranging from $10^{-10}$ to $10^{-5} \mathrm{M}$ in medium. Estradiol $\left(\mathrm{E}_{2}\right)$ at the concentration of $10^{-9} \mathrm{M}$ was used as the positive control. After $48-72 \mathrm{~h}, 20 \mu \mathrm{l}$ of MTT solution $(0.5 \mathrm{mg} / \mathrm{ml})$ were then added to each well and the mixture was incubated at $37^{\circ} \mathrm{C}$ for $4 \mathrm{~h}$ to allow the formation of formazan crystal. Culture medium was then replaced with equal volume of DMSO to dissolve the dark blue crystals. After shaking at room temperature for $10 \mathrm{~min}$ to ensure that all crystals were totally dissolved, absorbance was measured at $490 \mathrm{~nm}$ by a Bio-Rad ELISA reader (Bio-Rad, CA, USA).

Cell proliferation assay. Cell proliferation was determined by means of EdU assay (32). Osteoblasts $\left(2 \times 10^{4}\right.$ cells/well) were seeded in 24-well plates. Cells were incubated in full medium for $24 \mathrm{~h}$ before the $10^{-6}$ and $10^{-5} \mathrm{M}$ AST II was added for $48 \mathrm{~h} . \mathrm{E}_{2}$ at the concentration of $10^{-9} \mathrm{M}$ was used as the positive control. After incubated with EdU for $2 \mathrm{~h}$, EdU incorporation was performed on the primary osteoblastic cells. The cells are fixed with $4 \%$ paraformaldehyde for $30 \mathrm{~min}$ at room temperature. This is followed by Apollo for $30 \mathrm{~min}$ at room temperature. Then cells were incubated with Hoechst for $30 \mathrm{~min}$ at room temperature followed by PBS wash. Stained sections were examined under a fluorescence microscope (Nikon, Tokyo, Japan). Fluorescence signals were detected using a band-pass filter $555 \pm 15 \mathrm{~nm}$ for the $550 \mathrm{~nm}$ excitation. For each sample, approximately 200 cells were counted and the percentage of EdU-positive cells was calculated.

Measurement of alkaline phosphatase activity. ALP activity was determined by quantitative and staining examination. Osteoblasts cultured in 24-well plates with MEM containing $1 \%$ FBS, L-ascorbic acid $(50 \mu \mathrm{g} / \mathrm{ml})$ and $\beta$-glycerophosphate $(10 \mathrm{mM})$ in the presence or absence of agents $\left(10^{-7}-10^{-5} \mathrm{M}\right)$. $\mathrm{E}_{2}$ at the concentration of $10^{-9} \mathrm{M}$ was used as the positive control. After 1 to 7 days, cells were gently washed twice with PBS, then lysed with $0.2 \%$ Triton X-100 and the lysate was centrifuged at $14,000 \mathrm{x}$ g for $5 \mathrm{~min}$. The supernatant was collected for the measurement of ALP activity by ALP activity assay kit, and protein concentration were determined by BCA-protein assay kit, respectively (33).

Measurement of collagen I and osteocalcin levels. Collagen I and osteocalcin ELISA kits were used to detect collagen I and osteocalcin levels, respectively. Cells were treated with various concentrations $\left(10^{-7}-10^{-5}\right)$ of AST II for the indicated times (5-8 days). $E_{2}$ at the concentration of $10^{-9} \mathrm{M}$ was used as the positive control. According to the instructions, these samples were placed in 96-well microtiter plates coated with monoclonal detective antibodies and incubated for $2 \mathrm{~h}$ at room temperature. After removing unbound material with washing buffer, horseradish peroxidase conjugated streptavidin was added to bind to the antibodies. Horseradish peroxidase catalyzed the conversion of a chromogenic substrate (tetramethylbenzidine) to a colored solution, with color intensity in proportion to the amount of protein present in the sample. The absorbance of each well was measured at $450 \mathrm{~nm}$. Results are presented as the percentage of change of the activity compared with the untreated control.
Measurement of mineralized matrix. Mineralization in osteoblast cultures was determined by Alizarin Red-S (Aldrich, Milwaukee, WI, USA) staining. Osteoblasts were seeded in 24-well plates at a density of $2 \times 10^{5}$ cells/well. After 2 days of incubation, cells were washed twice with PBS solution, treated with or without AST II, and cultured with MEM containing $1 \%$ FBS, L-ascorbic acid $(50 \mu \mathrm{g} / \mathrm{ml})$ and $\beta$-glycerophosphate $(10 \mathrm{mM})$, and the medium were changed every 3 days. $E_{2}$ at the concentration of $10^{-9} \mathrm{M}$ was used as the positive control. After incubation with and without drugs for 14 days, cells were washed twice with PBS solution at room temperature, fixed with $70 \%$ ethanol for $1 \mathrm{~h}$, washed three times with distilled water, then stained with $40 \mathrm{mmol} / \mathrm{l}$ Alizarin Red-S (pH 4.2) for $15 \mathrm{~min}$ at room temperature. After removing the Alizarin Red-S solution, cultures were washed three times with deionized water and then cultured with PBS for another $15 \mathrm{~min}$. Images of the mineralized matrices were photographed using Nikon microscope. To quantify matrix mineralization, Alizarin Red-S stained cultures were incubated in $100 \mathrm{mmol} / \mathrm{l}$ cetylpyridinium chloride (Sigma) for $1 \mathrm{~h}$ to solubilize and release calcium-bound Alizarin Red-S into solution. The extracted stain was then transferred to a 96-well plates, and the absorbance of the released Alizarin Red-S was read on ELISA reader at $570 \mathrm{~nm}$ (33).

Assaying the levels of secreting BMP-2. Cells were treated with various concentrations $\left(10^{-7}, 10^{-6}, 10^{-5} \mathrm{M}\right)$ of AST II for $24 \mathrm{~h}$. The culture medium was collected and measured for BMP-2 using BMP-2 ELISA kits, followed the same steps as in section 2.7. Results are presented as the percentage of change of the activity compared with the untreated control.

Analysis for western blotting. To cells treated with or without agents for $24 \mathrm{~h}, 1 \mathrm{mmol} / \mathrm{l}$ PMSF was added and lysed by Nuclear and cytoplasmic protein extraction kit at $4^{\circ} \mathrm{C}$. The protein concentration was determined by BCA-protein assay kit. After obtaining the soluble fraction of nuclear and cytoplasmic protein, respectively, they were used for the western blotting. Equal amounts of protein were subjected to a $15 \%$ SDS-polyacrylamide gel electrophoresis. The protein was transferred to nitrocellulose membranes (Pall, NY, USA) using transfer buffer (50 mM Tris, $190 \mathrm{mM}$ glycin, and 10\% methanol) at $50 \mathrm{~V}$ for $2.5 \mathrm{~h}$. The membranes were incubated with blocking buffer $(0.05 \%$ Tween-20 and 5\% non-fat milk) for $12 \mathrm{~h}$ at $4^{\circ} \mathrm{C}$. After washing three times with PBS, the blot was incubated with primary antibody (rabbit polyclonal anti-Smad1/5/8, anti-phosphorylated Smad1/5/8, anti-p38, anti-phosphospecific p38, anti-ERK1/2, anti-phosphospecific ERK1/2, anti-JNK, anti-phosphospecific JNK, anti-Cbfa1, anti-Histone, $1 / 500$ dilution; mouse monoclonal anti- $\beta$-actin, $1 / 1,000$ dilution) for $12 \mathrm{~h}$ at $4^{\circ} \mathrm{C}$. Subsequently, the membranes were washed three times for $10 \mathrm{~min}$ with TBS buffer containing $0.05 \%$ Tween-20, and then incubated with anti-rabbit or anti-mouse secondary antibodies $(1: 5,000)$ for $1 \mathrm{~h}$ at room temperature. The membranes were washed three times for 10 min with TBS buffer, and once for 10 min with PBS. Detection was performed using the enhanced chemiluminescence western blotting detection system (Millipore, MA, USA) (34).

Statistical analysis. Data are expressed as the mean \pm SD. Statistical analyses were done by using the one-way ANOVA 
A

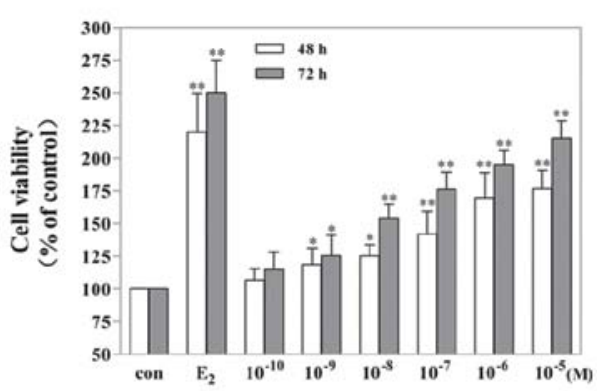

B
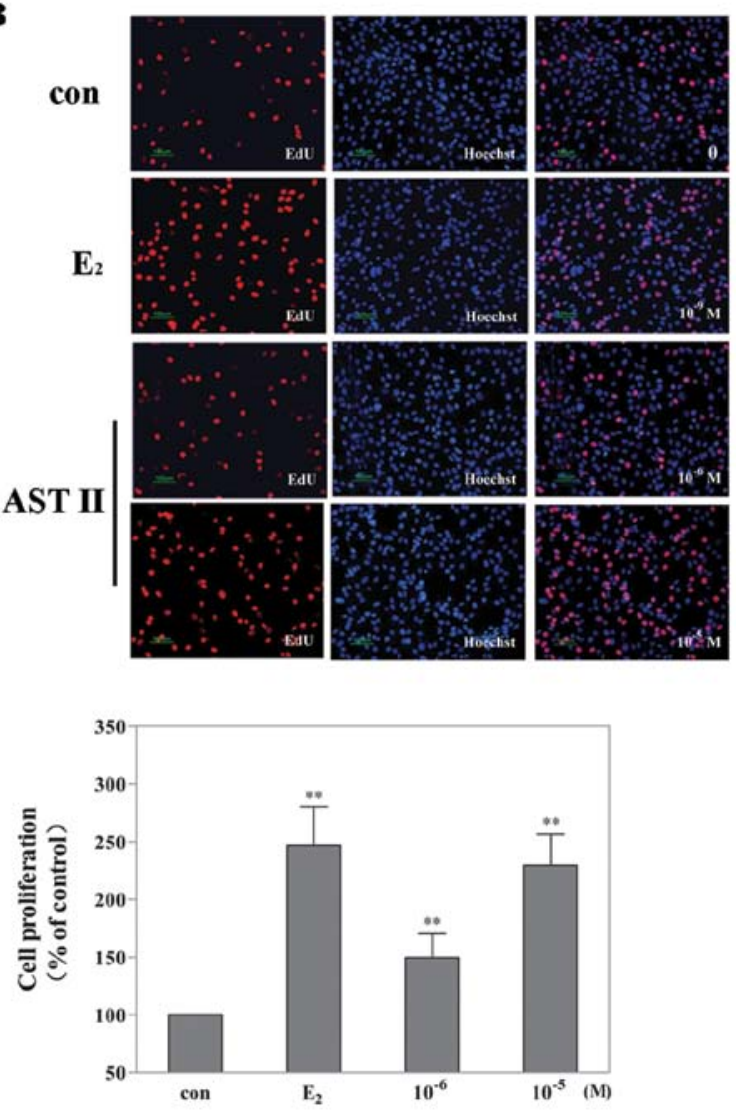

Figure 2. The effects of Astragaloside II (AST II) on the cell viability and proliferation of rat primary osteoblastic cells. Adherent cells that proliferated were incubated with different concentrations (M) of AST II for various time intervals. Cell viability and proliferation were evaluated by (A) the MTT and (B) EdU assay (x200), respectively. For each sample, approximately 200 cells were counted and the percentage of EdU-positive cells was calculated. Estradiol $\left(\mathrm{E}_{2}, 10^{-9} \mathrm{M}\right)$ was used as the positive control. Values are the mean \pm SD of three independent experiments. ${ }^{*} \mathrm{P}<0.05$ as compared with control; ${ }^{* *} \mathrm{P}<0.01$ as compared with control $(\mathrm{n}=5)$.

test to compare the different groups. A probability $\mathrm{P}$-value $<0.05$ was considered to be statistically significant.

\section{Results}

AST II promotes the viability of rat primary osteoblasts. We first determined the effect of AST II on the cell viability of rat primary osteoblasts by means of MTT assay. Our results showed that either treatment of osteoblasts with AST II $\left(10^{-10}\right.$ $10^{-5} \mathrm{M}$ ) or $\mathrm{E}_{2}$ (the positive control) for 48 and $72 \mathrm{~h}$, significantly promoted the cell viability in a concentration-dependent manner (Fig. 2A).
A

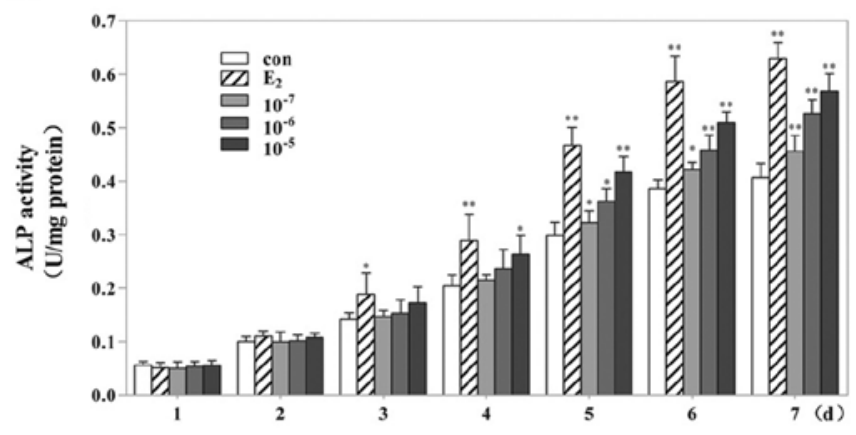

B

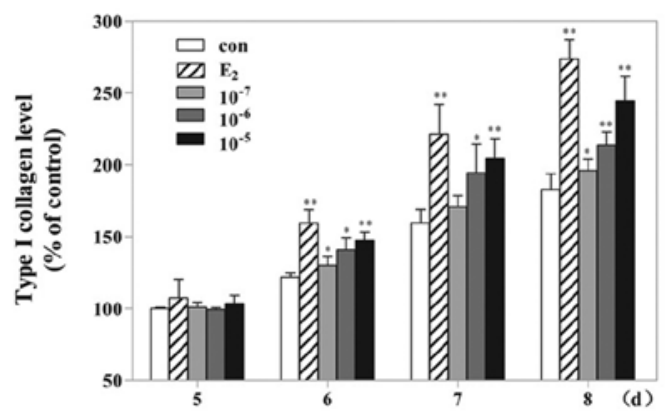

C

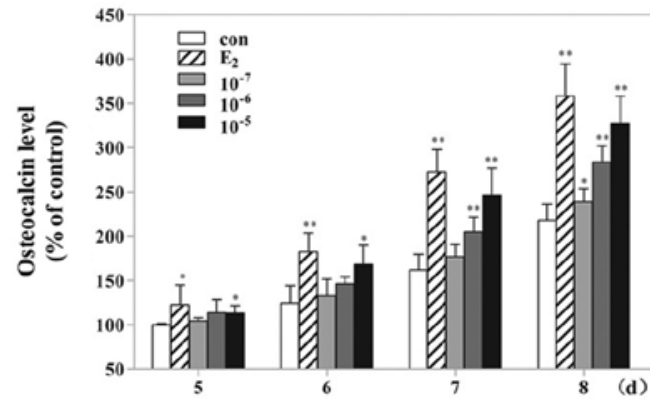

Figure 3. Enhancement of the differentiation of osteoblast by Astragaloside II (AST II). AST II increased (A) ALP activity, (B) type I collagen synthesis, and (C) osteocalcin production. Rat primary osteoblastic cells were treated with various concentrations (M) of AST II for various time intervals. ALP activity was assessed using a commercial ALP kit. The amount of type I collagen and osteocalcin in culture medium were assessed by type I collagen and osteocalcin ELISA kits. $\mathrm{E}_{2}\left(10^{-9} \mathrm{M}\right)$ was used as the positive control. ${ }^{*} \mathrm{P}<0.05$ as compared with control; ${ }^{* *} \mathrm{P}<0.01$ as compared with control $(\mathrm{n}=5)$. The values represent mean $\pm \mathrm{SD}$ of three independent experiments.

AST II enhances the proliferation of rat primary osteoblasts. The effect of AST II on the cell proliferation of rat primary osteoblasts was evaluated by means of EdU incorporation assay. The percentage of EdU-positive cells, indicating progression into $\mathrm{S}$-phase, was calculated. In the control group, $24 \%$ of the cells were EdU-positive, compared with $36 \%$ and $55 \%$ of the cells treated with $10^{-6}$ and $10^{-5}$ M AST II for $48 \mathrm{~h}$, and $59 \%$ with $10^{-9} \mathrm{M} \mathrm{E}_{2}$ (the positive control) (Fig. 2B).

AST II enhances the differentiation of rat primary osteoblasts. ALP activity is a phenotypic marker for the early differentiation of osteoblasts. We assessed the effect of AST II on the 

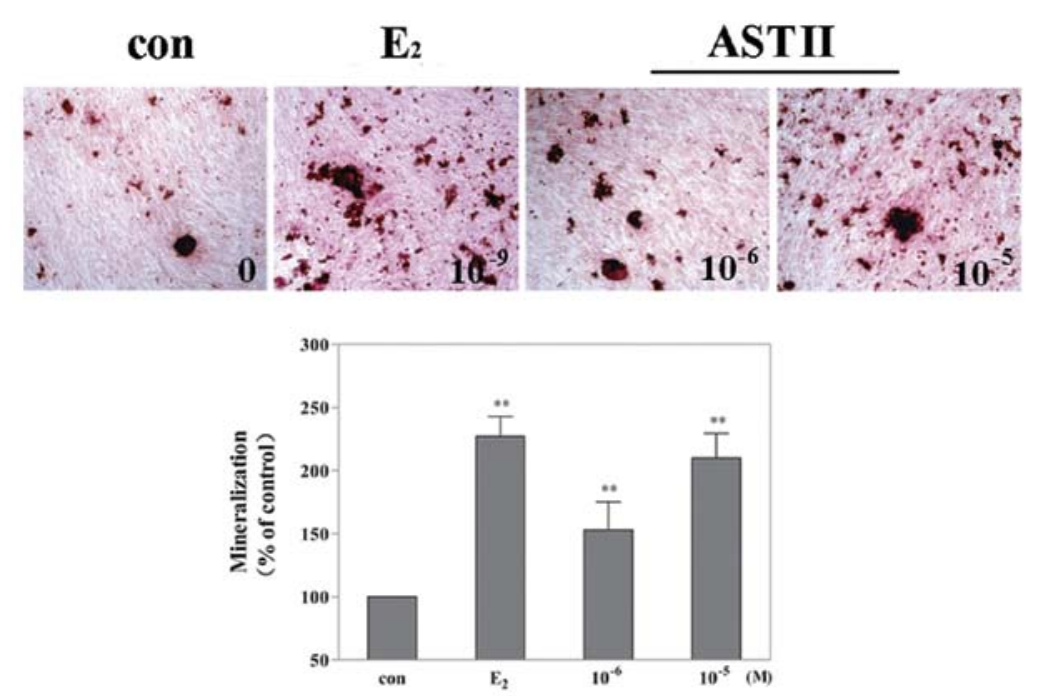

Figure 4. Astragaloside II (AST II) increased the mineralization of osteoblasts. Cells were cultured in 24-well plates and cultured in medium containing vitamin C $(50 \mu \mathrm{g} / \mathrm{ml})$ and $\beta$-glycerophosphate $(10 \mathrm{mM})$. The cells were treated with or without AST II $\left(10^{-6} \mathrm{M}\right.$ and $\left.10^{-5} \mathrm{M}\right)$ for 2 weeks. At the end of the experiment, the mineralized nodule formation was assessed by Alizarin Red-S staining (x200). The bound staining was washed with a solution of $100 \mathrm{mmol} / 1$ cetylpyridinium chloride and quantified using a Bio-Rad ELISA reader. $\mathrm{E}_{2}\left(10^{-9} \mathrm{M}\right)$ was used as the positive control. ${ }^{*} \mathrm{P}<0.05$ as compared with control; ${ }^{* *} \mathrm{P}<0.01$ as compared with control $(\mathrm{n}=5)$. Values are the mean $\pm \mathrm{SD}$ of three independent experiments.

activity ALP. The results showed that the first 3 days after treatment of AST II, the ALP activity in osteoblasts was not increased significantly. While from 4-7 days after treatment, the ALP activity was increased by AST II and $\mathrm{E}_{2}$ (the positive control) respectively, in a concentration-dependent manner (Fig. 3A).

The effect of AST II on the late differentiation of osteoblasts was also assessed by determining the production of osteocalcin and type I collagen proteins. As shown in Fig. 3B and C, the levels of type I collagen and osteocalcin proteins were increased by AST II and $\mathrm{E}_{2}$ (the positive control) respectively, in a concentration-dependent manner from 5-8 days after treatment.

AST II enhances the maturation of primary osteoblasts. The formation of calcified nodule is one of the markers of the osteoblastic maturation. The calcified nodules appeared bright red by Alizarin Red-S staining (Fig. 4). The results showed that the formation of bone nodules was increased in the osteoblasts treated with AST II at various concentrations for 2 weeks. AST II at $10^{-6}$ and $10^{-5} \mathrm{M}$ was able to stimulate approximately 153 and $210 \%$ increases in the formation of calcified nodules compared to the control. The positive control $\mathrm{E}_{2}$ at $10^{-9} \mathrm{M}$ could stimulate approximately $227 \%$ increase compared to the control.

BMP-2 mediates AST II-induced upregulation of differentiation in primary osteoblasts. It has been demonstrated that BMP-2 plays an important role in the process of bone formation (10). To confirm whether the levels of secreting BMP-2 expression were influenced by the presence of AST II, we examined the expression of the secreting BMP-2 in the presence and absence of AST II using BMP-2 ELISA kits. The results indicated that AST II $\left(10^{-7}, 10^{-6}, 10^{-5} \mathrm{M}\right)$ significantly increased the secreting BMP-2 protein levels in a concentration-dependent manner after $24 \mathrm{~h}$ of treatment (Fig. 5A).
To further examine the role of BMP-2 in AST II-induced cell proliferation and upregulation of differentiation, osteoblasts were pretreated with BMP-2 inhibitor, $100 \mathrm{ng} / \mathrm{ml}$ noggin protein for $2 \mathrm{~h}$ /day, then $10^{-5} \mathrm{M}$ AST II was added at the indicated time. Noggin directly binds to BMP-2, thereby preventing its interaction with BMP receptor (35). Addition of purified noggin protein did not change cell viability and ALP activity. In contrast, concurrent treatment with noggin did not change cell viability, but significantly diminished the AST II-induced ALP activity (Fig. 6A). Therefore, AST II-enhanced cell differentiation may be operated by a BMP-2-dependent pathway. It seemed that AST II-enhanced cell proliferation was not operated by BMP-2.

BMP-2 is involved in the activation of Smad1/5/8, ERK and p38 in AST II treated primary osteoblasts. Binding of BMP-2 to $\mathrm{BMP}$ receptor induces receptor heteromeric complexes and subsequently activates SMADs or MAPKs by phosphorylation $(11,12)$. We assessed the activation of Smad1/5/8, p38, ERK1/2 and JNK MAPKs in AST II treated cells. As shown in Fig. 5B, treatment with AST II did not affect the expression levels of unphosphorylated Smad1/5/8, p38, ERK1/2 and JNK, but did increase the levels of phospho-Smad1/5/8, phospho-p38 and phospho-ERK1/2 after $24 \mathrm{~h}$ exposure of osteoblasts to AST II. However, AST II did not significantly affect the level of phospho-JNK.

To determine the role of BMP-2 in AST II induced activation of Smad1/5/8, p38 and ERK1/2 in osteoblasts, osteoblast were pretreated with noggin for $2 \mathrm{~h}$, and then co-treated with $10^{-5} \mathrm{M}$ AST II for $24 \mathrm{~h}$. Results showed that noggin pretreatment abrogated the activation of p38 and Smad1/5/8 induced by AST II (Fig. 6B). However, noggin pretreatment had a lesser effect on ERK1/2 (Fig. 6B). It indicates that BMP-2 signaling is the main path participating in the activation of $\mathrm{p} 38$ and Smad1/5/8, but only partly involved in the activation of ERK1/2 induced by AST II. 
A

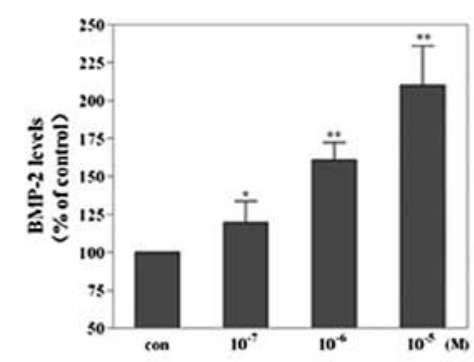

B
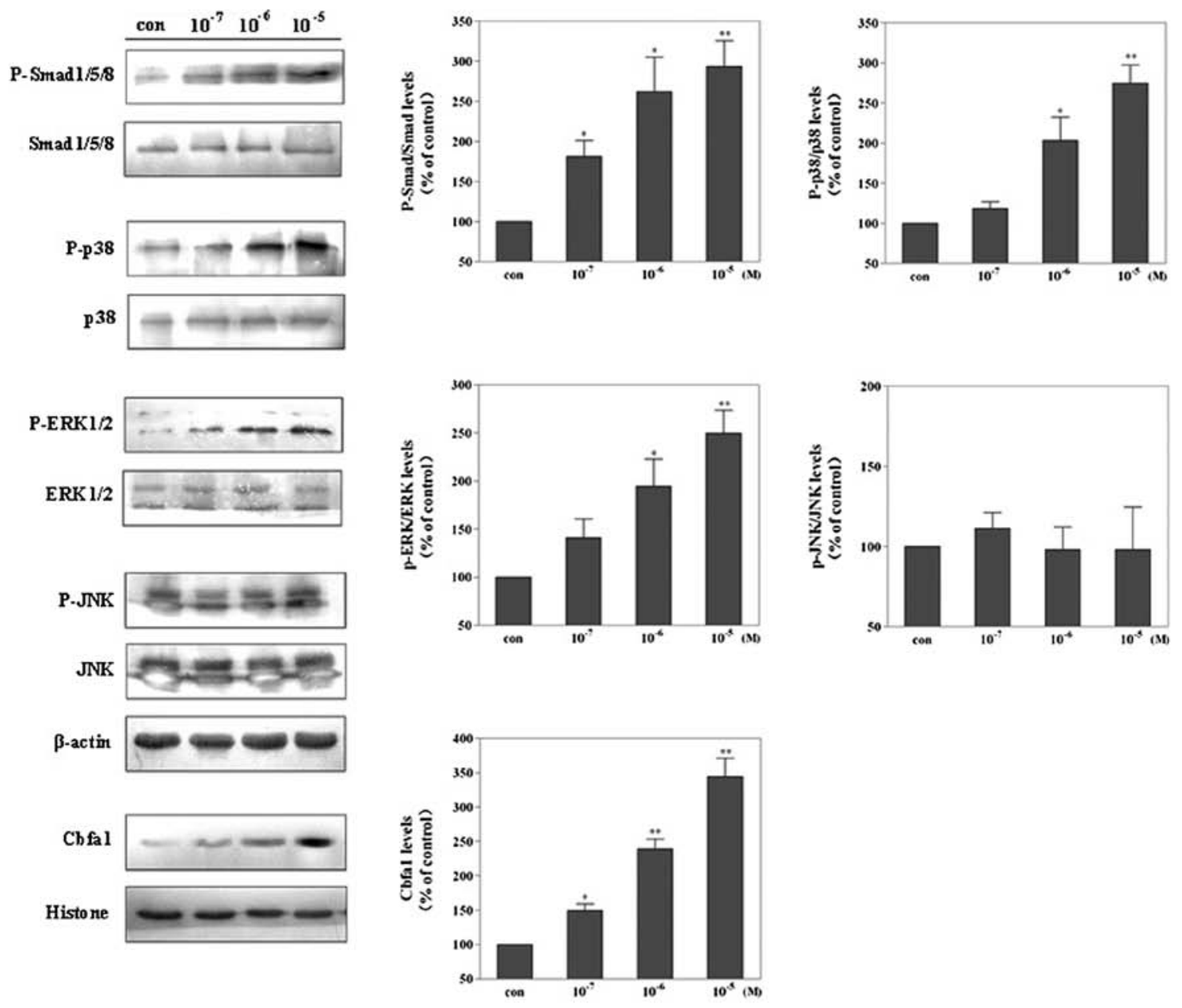

Figure 5. The effects of Astragaloside II (AST II) on the protein levels of (A) BMP-2, (B) Smad1/5/8, p38, ERK1/2, JNK and Cbfa1 in osteoblasts. The protein level of BMP-2 was assessed by BMP-2 ELISA kit. Western blot analysis of Smad1/5/8, p38, ERK1/2, JNK and Cbfa1 expressions after treated with $10^{-7}, 10^{-6}$ and $10^{-5} \mathrm{M}$ of AST II in osteoblastic cells for $24 \mathrm{~h}$. 'Quantity one' of Bio-Rad was used to analyze the results of western blotting. "P $<0.05$ as compared with control; ${ }^{* *} \mathrm{P}<0.01$ as compared with control $(\mathrm{n}=3)$. The values represent mean $\pm \mathrm{SD}$ of three independent experiments.

BMP-2 is necessary for the increasing expression of Cbfal in AST II treated osteoblasts. Cbfa1 is an essential transcription factor required for osteoblast differentiation (13). After treatment with AST II for $24 \mathrm{~h}$, protein of Cbfa1 in primary osteoblasts was obviously increased (Fig. 5B). To determine the role of BMP-2 in AST II-induced activation of Cbfa1 in osteoblasts, osteoblast were pretreated with or without noggin for $2 \mathrm{~h}$, and then co-treated with $10^{-7} \mathrm{M}$ AST II for $24 \mathrm{~h}$. When osteoblasts were incubated with AST II in the presence of noggin, the stimulatory effect of AST II on Cbfa1 protein was markedly reduced (Fig. 6B). These results suggest that BMP-2 signaling is necessary for the increasing expression of $\mathrm{Cbfa} 1$ in AST II-treated primary osteoblasts.

\section{Discussion}

The formation of bone involves a complex series of events, including the proliferation and differentiation of osteoblasts and result eventually in the formation of a mineralized extracellular matrix. Since new bone formation is primarily a function of the osteoblast, agents that regulate bone formation act by either increasing the proliferation of cells of the osteoblastic lineage or inducing differentiation and mineralization of the osteoblasts (36). In this research, we examined the effects of AST II on the proliferation, differentiation and mineralization of primary rat osteoblasts. The results show that AST II can stimulate the proliferation, enhance the alkaline phosphatase 
A

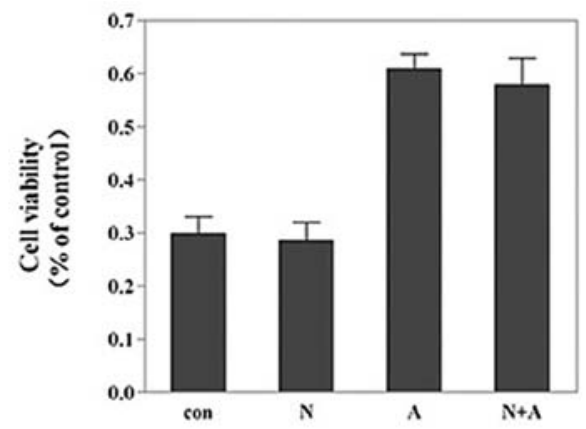

B
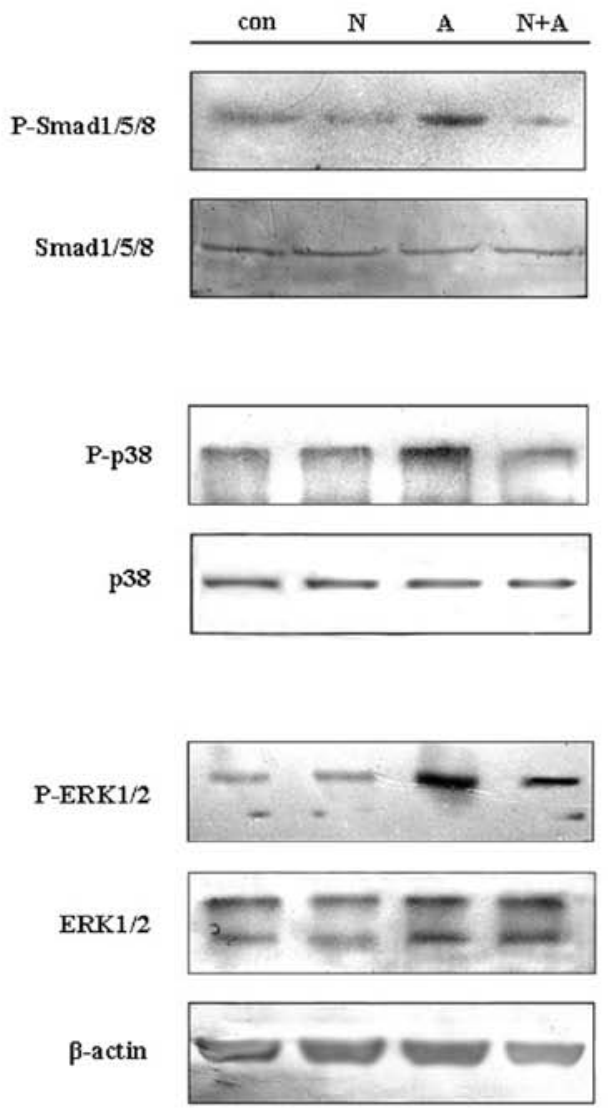

Cbfa1

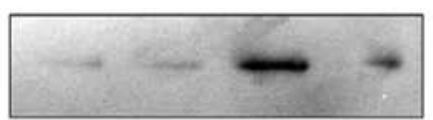

Histone

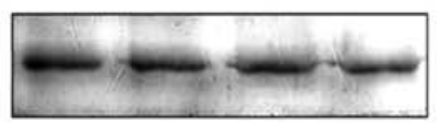

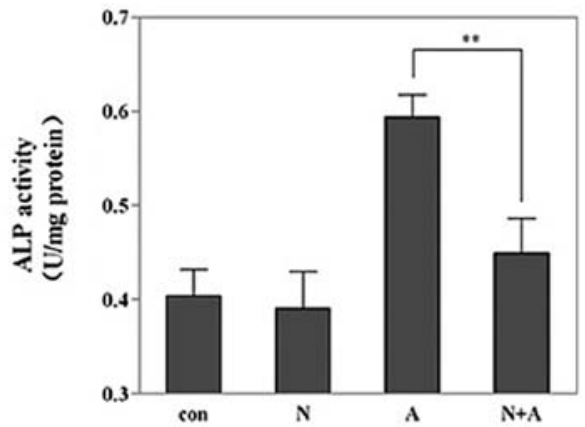
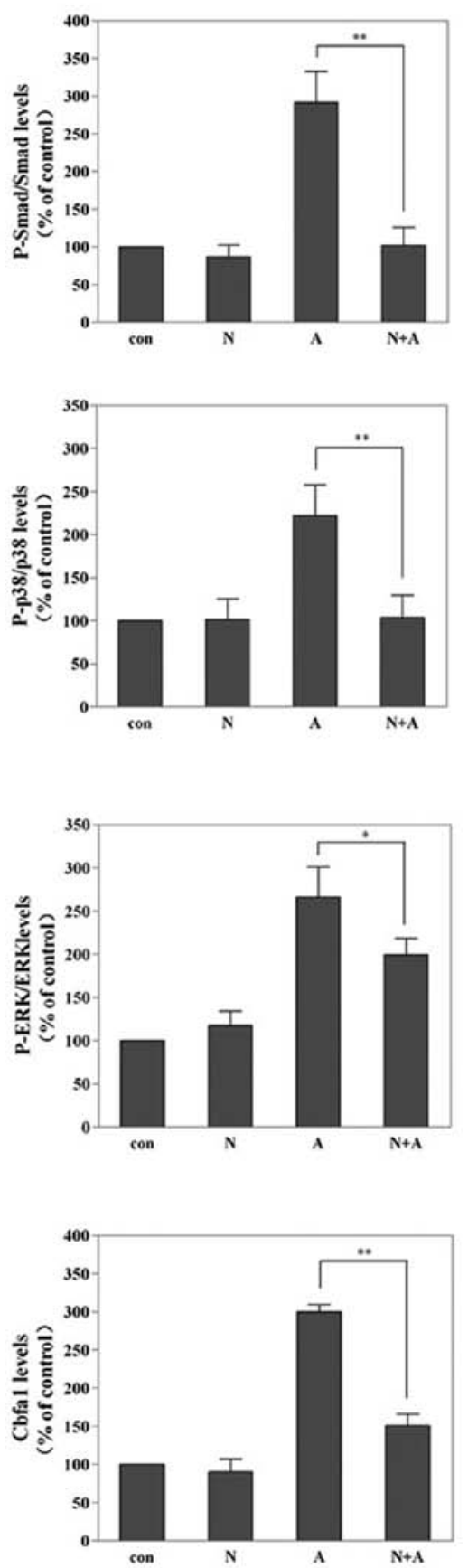

Figure 6. The role of BMP-2 in upregulation of osteoblastic activity by Astragaloside II (AST II) in osteoblasts. (A) Noggin inhibited the induction of AST II on ALP upregulation, but did not affect the induction of AST II on cell viability. (B) Noggin also inhibited the induction of AST II on Smad1/5/8, p38, ERK1/2, Cbfa1 stimulation. (A), Cells were pretreated with or without $100 \mathrm{ng} / \mathrm{ml}$ noggin for $2 \mathrm{~h} /$ day, and then $10^{-5} \mathrm{M}$ AST II was added. After 3 days, cell viability was evaluated by the MTT assay. ALP activity was assessed using ALP kit after 7 days. (B), Cells were incubated for $2 \mathrm{~h}$ in the presence or absence of noggin, and then $10^{-5} \mathrm{M}$ AST II was added and incubated for $24 \mathrm{~h}$. The levels of Smad1/5/8, p38, ERK1/2 and Cbfa1 were determined by western blot analysis. Each value is the mean \pm SD of three independent experiments. N, Noggin; A, Astragaloside II. 
activity (an early stage marker of differentiation), increase the levels of type I collagen and osteocalcin proteins (late stage markers of differentiation), and promote the formation of bone nodule (a marker of mineralization) in primary rat osteoblasts. Although $\mathrm{E}_{2}$ as the positive control shows slightly higher effects on the proliferation, differentiation and mineralization of osteoblasts than AST II, the side effects of the long-term use of estrogen, such as a higher incidence of breast carcinoma, endometrial cancer, and cardiovascular disease (21), could not be ignored. Thus, AST II could affect various levels of differentiation process of osteoblast, from early to late stages, which indicated that AST II may be one of the anti-osteoporosis active ingredients of Astragalus.

Then we focused on the signaling pathway of AST II in inducing maturation of osteoblasts. BMPs play an important role in the formation and remodeling of bone by stimulating the differentiation of osteoblast cells $(10,31)$. In the BMP subfamily, BMP-2, which is synthesized and secreted into the extracellular by osteoblasts, has demonstrated a strong osteo-inductive capacity in vivo and in vitro $(6,7)$. The action of BMPs is mediated by hetero-tetrameric serine/threonine kinase receptors and the downstream transcription factors Smad1/5/8. Upon phosphorylation by type I receptors, Smad1/5/8 form complexes with Smad4, translocate into the nucleus and regulate transcription of target genes associated with differentiation (9). This study indicates that the production of secreting BMP-2 was increased in AST II-treated cells. Also, phosphorylation of Smad1/5/8 is enhanced in AST II-treated osteoblasts.

In addition to Smad activation, BMP-2 can activate Smadindependent pathways, such as MAPK signaling $(37,38)$. BMP-2 can stimulate Ras activity and as a consequence, two MAPKs, ERK and P38 (37,39). P38 and ERK MAPK activation is essential in the BMP-2 upregulation of alkaline phosphatase, type I collagen, osteocalcin, and osteopontin (38). This study observed an increase in p38 and ERK activity in AST II treated cells. These data suggest that activation of p38 and ERK may play an important role on the increase of BMP-2 expression and the cell differentiation by AST II in primary rat osteoblasts. However, the actual mechanism by which AST II operates the two different MAPK signals to regulate different phases of osteoblast differentiation requires further investigation.

The transcription factor, Cbfa1, is a key mediator, required for osteoblastic cell differentiation and ossification (40). It is crucial for regulating expression of bone matrix proteins, such as osteocalcin, type I collagen, osteopontin, and bone sialoprotein (41). It has been reported that both the Smad and MAPK pathways are essential components of the BMP signaling during osteoblast differentiation $(11,14-16,42)$ and for Cbfa1 induction (18). Our study indicates that treating osteoblasts with AST II can promote the expression of Cbfa1 protein, which indicates that AST II may exert its action on bone anabolism through modulating Cbfa1.

What is the role of BMP-2 in the AST II causing a series of changes on osteoblast? As we found in this study, the bone morphogenetic protein antagonist noggin exhibits a suppressive effect against AST II-mediated ALP upregulation, which indicates that the BMP-2 signaling system plays an important role in promoting AST II-mediated cell differentiation. However, it seems that AST II-enhanced cell proliferation is not operated by BMP-2. Furthermore, suppression of BMP-2 signaling by cotreating noggin abrogates Smad1/5/8 and p38 activation in AST II-treated cells, which suggests that the BMP-2 pathway participated in the activation of Smad1/5/8 and p38. However, co-treatment of osteoblast cells with noggin and AST II only partly blocks the activation of ERK1/2. This suggests that the activation of ERK1/2 by AST II may be simultaneously through both BMP-2-dependent and -independent pathways. The increase in Cbfal protein by AST II can be prevented by the BMP inhibitor noggin, which demonstrates an involvement of the BMP-2 pathway in the stimulatory effect of AST II on Cbfa1.

Although Radix Astragalus has been used as a traditional Chinese medicine for anti-osteoporosis, it has been used only as healthcare products in the world, because its active ingredients and mechanism of the action are not very clear. The significance for our finding is that AST II as a novel phyto-saponin, promoting osteogenesis activity in vitro, which provides scientific evidence and support for the belief in traditional Chinese medicine that Radix Astragalus has the ability to strengthen bones and has long been used to treat osteoporosis. This study indicates that in vitro AST II is a bone anabolic agent that may exert its osteogenic effects through the induction of BMP-2, Smad-dependent (Smad1/5/8) and -independent (p38 and ERK1/2) signal pathways. All of aforementioned effects may contribute to the induction of osteoblasts proliferation and differentiation.

In conclusion, our study in vitro indicates that AST II may become a novel phyto-saponin that is beneficial for stimulating the osteoblastic activity resulting in bone formation. As a phyto-saponin of Radix Astragalus, AST II may be a good candidate for promoting bone health, which has not been recognized or reported previously. However, in vivo studies are required.

\section{Acknowledgements}

This study was supported by the Doctorate Foundation of Northwestern Polytechnical University (grant no. CX201024), the Ministry of Education Fund for Doctoral Students Newcomer Awards of China, and the National Natural Science Foundation of China (grant no. 81073037).

\section{References}

1. Manolagas SC and Jilka RL: Bone marrow, cytokines, and bone remodeling. Emerging insights into the pathophysiology of osteoporosis. N Engl J Med 332: 305-311, 1995.

2. Teitelbaum SL: Osteoclasts, integrins, and osteoporosis. J Bone Miner Metab 18: 344-349, 2000.

3. Reddi AH: Bone and cartilage differentiation. Curr Opin Genet Dev 4: 737-744, 1994.

4. Sakou T, Onishi T, Yamamoto T, Nagamine T, Sampath T and Ten Dijke P: Localization of Smads, the TGF-beta family intracellular signaling components during endochondral ossification. J Bone Miner Res 14: 1145-1152, 1999.

5. Tang CH, Yang RS, Huang TH, Liu SH and Fu WM: Enhancement of fibronectin fibrillogenesis and bone formation by basic fibroblast growth factor via protein kinase C-dependent pathway in rat osteoblasts. Mol Pharmacol 66: 440-449, 2004.

6. Jia TL, Wang HZ, Xie LP, Wang XY and Zhang RQ: Daidzein enhances osteoblast growth that may be mediated by increased bone morphogenetic protein (BMP) production. Biochem Pharmacol 65: 709-715, 2003. 
7. Takuwa Y, Ohse C, Wang EA, Wozney JM and Yamashita K : Bone morphogenetic protein-2 stimulates alkaline phosphatase activity and collagen synthesis in cultured osteoblastic cells, MC3T3-E1. Biochem Biophys Res Commun 174: 96-101, 1991.

8. Derynck R and Zhang YE: Smad-dependent and Smadindependent pathways in TGF-beta family signalling. Nature 425: 577-584, 2003.

9. Nohe A, Keating E, Knaus P and Petersen NO: Signal transduction of bone morphogenetic protein receptors. Cell Signal 16: 291-299, 2004

10. Sykaras N and Opperman LA: Bone morphogenetic proteins (BMPs): how do they function and what can they offer the clinician? J Oral Sci 45: 57-73, 2003.

11. Gallea S, Lallemand F, Atfi A, et al: Activation of mitogenactivated protein kinase cascades is involved in regulation of bone morphogenetic protein-2-induced osteoblast differentiation in pluripotent C2C12 cells. Bone 28: 491-498, 2001.

12. Guicheux J, Lemonnier J, Ghayor C, Suzuki A, Palmer G and Caverzasio J: Activation of $\mathrm{p} 38$ mitogen-activated protein kinase and c-Jun-NH2-terminal kinase by BMP-2 and their implication in the stimulation of osteoblastic cell differentiation. J Bone Miner Res 18: 2060-2068, 2003.

13. Sakai S, Tamura M, Mishima H, Kojima H and Uemura T: Bone regeneration induced by adenoviral vectors carrying til-1/Cbfa 1 genes implanted with biodegradable porous materials in animal models of osteonecrosis of the femoral head. J Tissue Eng Regen Med 2: 164-167, 2008

14. Derynck R, Akhurst RJ and Balmain A: TGF-beta signaling in tumor suppression and cancer progression. Nat Genet 29: 117-129, 2001.

15. Fujii M, Takeda K, Imamura T, et al: Roles of bone morphogenetic protein type I receptors and Smad proteins in osteoblast and chondroblast differentiation. Mol Biol Cell 10: 3801-3813, 1999.

16. Yamamoto N, Akiyama S, Katagiri T, Namiki M, Kurokawa T and Suda T: Smad1 and smad5 act downstream of intracellular signalings of BMP-2 that inhibits myogenic differentiation and induces osteoblast differentiation in $\mathrm{C} 2 \mathrm{C} 12$ myoblasts. Biochem Biophys Res Commun 238: 574-580, 1997.

17. Hsieh TP, Sheu SY, Sun JS, Chen MH and Liu MH: Icariin isolated from Epimedium pubescens regulates osteoblasts anabolism through BMP-2, SMAD4, and Cbfa1 expression. Phytomedicine 17: 414-423, 2010 .

18. Lee KS, Hong SH and Bae SC: Both the Smad and p38 MAPK pathways play a crucial role in Run 2 expression following induction by transforming growth factor-beta and bone morphogenetic protein. Oncogene 21: 7156-7163, 2002.

19. Chen PY, Sun JS, Tsuang YH, Chen MH, Weng PW and Lin FH Simvastatin promotes osteoblast viability and differentiation via Ras/Smad/Erk/BMP-2 signaling pathway. Nutr Res 30: 191-199, 2010.

20. Li X, Cui Q, Kao C, Wang GJ and Balian G: Lovastatin inhibits adipogenic and stimulates osteogenic differentiation by suppressing PPARgamma 2 and increasing Cbfa1/Runx 2 expression in bone marrow mesenchymal cell cultures. Bone 33: 652-659, 2003.

21. Davison S and Davis SR: Hormone replacement therapy: current controversies. Clin Endocrinol (Oxf) 58: 249-261, 2003.

22. Cassidy A: Dietary phytoestrogens and bone health. $\mathrm{J} \mathrm{Br}$ Menopause Soc 9: 17-21, 2003.

23. Pereira JV and Filho J: Plant and plant-derived compounds employed in prevention of the osteoporosis. Acta Farm Bonaerense 21: $223-234,2002$.
24. Zhou ZK, Zeng HB, Qin JJ and Chen C: Treatment of postmenopausal osteoporosis by huangqi sanxian tang: a clinical observation of 30 cases. New J Tradit Chin Med 38: 40-41, 2006.

25. Wegiel B and Persson JL: Effect of a novel botanical agent Drynol Cibotin on human osteoblast cells and implications for osteoporosis: promotion of cell growth, calcium uptake and collagen production. Phytother Res 24 (Suppl 2): S139-S147, 2010.

26. Sassa S, Sakamoto S, Zhou YF, Mori T, Kikuchi T and Shinoda H: Preventive effects of a Chinese herbal medicine, hochu-ekki-to, on bone loss in ovariectomized rats. In Vivo 15: 25-28, 2001.

27. Xu CJ, Jian XC and Cheng HQ: Influence of astragalus membranaceus injection on the proliferation of rabbit bone marrow stromal cells and differentiation to osteoblast. Zhong Nan Da Xue Xue Bao Yi Xue Ban 29: 489-491, 2004 (In Chinese).

28. Kim C, Ha H, Lee JH, Kim JS, Song K and Park SW: Herbal extract prevents bone loss in ovariectomized rats. Arch Pharm Res 26: 917-924, 2003

29. Li XD, Wang JS, Chang B, et al: Panax notoginseng saponins promotes proliferation and osteogenic differentiation of rat bone marrow stromal cells. J Ethnopharmacol 134: 268-274, 2011.

30. Shen Y, Li YQ, Li SP, Ma L, Ding LJ and Ji H: Alleviation of ovariectomy-induced osteoporosis in rats by Panax notoginseng saponins. J Nat Med 64: 336-345, 2010.

31. Tang $\mathrm{CH}$, Yang RS, Liou HC and Fu WM: Enhancement of fibronectin synthesis and fibrillogenesis by BMP-4 in cultured rat osteoblast. J Bone Miner Res 18: 502-511, 2003.

32. Cappella P, Gasparri F, Pulici M and Moll J: A novel method based on click chemistry, which overcomes limitations of cell cycle analysis by classical determination of BrdU incorporation, allowing multiplex antibody staining. Cytometry A 73: 626-636, 2008.

33. Yang RS, Lin WL, Chen YZ, et al: Regulation by ultrasound treatment on the integrin expression and differentiation of osteoblasts. Bone 36: 276-283, 2005.

34. Niu Y, Li Y, Huang H, et al: Asperosaponin VI, A Saponin Component from Dipsacus asper Wall, induces Osteoblast Differentiation through Bone Morphogenetic Protein-2/p38 and Extracellular Signal-regulated Kinase 1/2 Pathway. Phytother Res 25: 1700-1706, 2011

35. Hallahan AR, Pritchard JI, Chandraratna RA, et al: BMP-2 mediates retinoid-induced apoptosis in medulloblastoma cells through a paracrine effect. Nat Med 9: 1033-1038, 2003.

36. Lane NE and Kelman A: A review of anabolic therapies for osteoporosis. Arthritis Res Ther 5: 214-222, 2003.

37. Attisano L and Wrana JL: Signal transduction by the TGF-beta superfamily. Science 296: 1646-1647, 2002.

38. Lai CF and Cheng SL: Signal transductions induced by bone morphogenetic protein- 2 and transforming growth factor-beta in normal human osteoblastic cells. J Biol Chem 277: 15514-15522, 2002.

39. Weston CR, Lambright DG and Davis RJ: Signal transduction. MAP kinase signaling specificity. Science 296: 2345-2347, 2002.

40. Lian JB, Javed A, Zaidi SK, et al: Regulatory controls for osteoblast growth and differentiation: role of Runx/Cbfa/AML factors. Crit Rev Eukaryot Gene Expr 14: 1-41, 2004

41. Ducy P, Starbuck M, Priemel M, et al: A Cbfa1-dependent genetic pathway controls bone formation beyond embryonic development. Genes Dev 13: 1025-1036, 1999.

42. Nishimura R, Kato Y, Chen D, Harris SE, Mundy GR and Yoneda T: Smad5 and DPC4 are key molecules in mediating BMP-2-induced osteoblastic differentiation of the pluripotent mesenchymal precursor cell line C2C12. J Biol Chem 273: 1872-1879, 1998. 